\title{
Invited Editorial: “The Head and Neck Reconstructive Surgery National Surgical Quality Improvement Program (NSQIP): Evaluating Unplanned Returns to the Operating Room" by Tam $S$ et al.
}

\author{
Matthew M. Hanasono, MD ${ }^{1}$ \\ The University of Texas MD Anderson Cancer Center, Houston, TX
}

Tam et al. ${ }^{1}$ examined 467 patients undergoing head and neck cancer resection with immediate microvascular free flap reconstruction for factors associated with an unplanned return to the operating room (URTOR). In their multivariate analysis, the authors found that coagulopathy and use of alcohol were associated with URTOR. The novelty of their study compared with most prior studies of complications after head and neck oncologic surgery and free flap reconstruction is that their analysis was based on a modified version of the American College of Surgeons (ACS) National Surgical Quality Improvement Program (NSQIP) database. The strength of the ACS NSQIP methodology is that the data are prospectively collected by specially trained surgical clinical reviewers who record pre-, intra-, and postoperative variables as well as 30-day outcomes.

Studies based on prospectively collected data should have a higher degree of fidelity than those based on retrospective data because they eliminate recall bias. In recent years, many studies have used the ACS NSQIP database to identify risk factors for URTOR or overall complications after various surgical procedures. However, despite highquality data collection, the ACS NSQIP database does have limitations. ${ }^{2}$ One limitation is that although maintaining prospective databases greatly facilitates clinical outcomes research, it can be time-consuming and expensive. Because

(C) Society of Surgical Oncology 2019

First Received: 21 June 2019;

Published Online: 17 September 2019

M. M. Hanasono, MD

e-mail: mhanasono@mdanderson.org of this, participation in NSQIP is not feasible for every clinical practice, especially smaller institutions and individual practices. Consequently, the results may not be translatable to all practice settings.

Probably the biggest limitation, however, is that important procedure- or disease-specific data are not collected by the ACS NSQIP, which was originally designed to track complications after general and vascular surgery procedures. Without a higher degree of granularity, studies using the ACS NSQIP may not be particularly helpful in improving surgical quality in terms of reducing specific complications and identifying pertinent risk factors in other disciplines (e.g., surgical subspecialties such as head and neck surgery and plastic surgery). Tam et al. ${ }^{1}$ have attempted to address this limitation by developing a novel database using the NSQIP platform that specifically collects variables relevant to head and neck oncologic surgery and free flap reconstruction, which they have described previously. ${ }^{3}$ In head and neck free flap surgery, the most important complication probably is free flap loss due to the extensive amount of surgery that free flaps entail and the implications that a failure to perform the optimal reconstruction has for patient function and appearance.

Orocutaneous or pharyngocutaneous fistula formation is another site-specific complication that occurs with some frequency and carries with it potential for substantial morbidity. Variables that the Head and Neck Reconstructive Surgery NSQIP database captures include information on the anatomic subsite of disease, flap type, and tracheostomy and feeding tube usage, all of which can potentially influence outcomes for this particular type of surgery. Such enhancements are critical to studying outcomes of interest, and their absence from the original 
version of the NSQIP likely is the reason the ACS NQIP risk calculator is demonstrated to be of poor predictive value in head and neck cancer surgery, as noted by Tam et al. ${ }^{1}$

Due to the findings of the current study, it seems logical to conclude that the coagulopathic conditions and the anticoagulants were associated with increased hematoma formation. ${ }^{4}$ Quality improvement should therefore focus on appropriate reversal of the coagulopathic state when possible. However, the potential development of a hematoma in some patients may have to be accepted given the risks of complete discontinuation or reversal of anticoagulation. These patients include those with preexisting venous thromboembolism, a history of cardiac stents, or a history of prior or intraoperative free flap pedicle thrombosis, which was likely in the many of the cases in the current study.

Alcoholism seems to be indirectly related to the occurrence of surgical-site infection. Furthermore, the authors found alcoholism to be a statistically significant risk factor due to the action of associated confounders (variables that are not part of the real association between exposure and outcome), in contrast to other studies, which have not frequently found alcoholism to be a risk factor for surgical complications. In fact, a disadvantage of all cohort studies is that they cannot truly establish causal effects. ${ }^{5}$ The possibility always exists that any associations identified in a cohort study may be explained by confounding variables that differ between subjects with and those without the potential risk factor that also have an association with the outcome studied. Even if alcohol abuse directly and significantly increases the risk of infection, this risk also may have to be accepted for many patients because surgery for active head and neck cancer usually cannot be delayed for satisfactory treatment because the tumor has the potential to grow and spread distantly in a relatively short time.

Other variables, previously identified as risk factors in other studies, such as prior irradiation, tobacco use, preoperative weight loss, and diabetes mellitus, did not seem to have an effect. ${ }^{6}$ This has important implications in terms of setting benchmarks for surgical quality and risk stratification. Although it may mean that these factors do not have a significant impact on the occurrence of complications, an alternate explanation is possible. It may be that, because they are well-known risk factors, steps already have been taken to prevent expected complications.
Individual providers and low-volume institutions may not have the experience necessary to prevent complications specific to those resulting from these factors and may not anticipate them because they are not shown to be associated with increased risk. If third-party reimbursement becomes dependent on meeting benchmarked norms, highvolume institutions with such experience may not be compensated appropriately for the extra time, expertise, and resources needed to avoid or minimize complications related to these factors. For example, prior radiotherapy was not found to be a significant risk factor for URTOR $(p=0.476)$ in the current study. However, experience dictates that performing head and neck resection and free flap reconstruction in the irradiated patient often is more taxing and time-consuming, mandating greater surgical expertise and greater postoperative vigilance to achieve complication-free results. Therefore, clinicians should interpret the lack of statistical significance of many potential risk factors with caution, remembering that the null hypothesis can never be proven true.

\section{REFERENCES}

1. Tam S, Weber RS, Liu J, Ting J, Hanson S, Lewis CM. The head and neck reconstructive surgery national surgical quality improvement program (NSQIP): evaluating unplanned returns to the operating room. Ann Surg Oncol. 2019. https://doi.org/10.1245/ s10434-019-07679-z.

2. Sippel RS, Chen H. Limitations of the ACS NSQIP in thyroid surgery. Ann Surg Oncol. 2011;18:3529-30.

3. Lewis CM, Aloia TA, Shi W, et al. Development and feasibility of a specialty-specific national surgical quality improvement program (NSQIP): the head and neck reconstructive surgery NSQIP. JAMA Otolaryngol Head Neck Surg. 2016;142:321-7.

4. Liu J, Shi Q, Yang S, Liu B, Guo B, Xu J. Does postoperative anticoagulation therapy lead to a higher success rate for microvascular free-tissue transfer in the head and neck? A systematic review and meta-analysis. J Reconstr Microsurg. 2018;34:87-94.

5. Euser AM, Zoccali C, Jager KJ, Dekker F. Cohort studies: prospective versus retrospective. Nephron Clin Pract. 2009;113:c214-7.

6. Ishimaru $M$, Ono $S$, Suuzuki $S$, et al. Risk factors for free flap failure in 2846 patients with head and neck cancer: a national database study in Japan. J Oral Maxillofac Surg. 2016;1265-70.

Publisher's Note Springer Nature remains neutral with regard to jurisdictional claims in published maps and institutional affiliations. 